\title{
Effect of Nanostructuring on the Reactivity of Zirconia: A DFT+U Study of Au Atom Adsorption
}

\author{
Antonio Ruiz Puigdollers ${ }^{\mathrm{a}}$, Francesc Illas ${ }^{\mathrm{b}}$, Gianfranco Pacchioni ${ }^{\mathrm{a},{ }^{,}}$ \\ ${ }^{a}$ Dipartimento di Scienza dei Materiali, Università di Milano-Bicocca. Via R. Cozzi 55, 20125 Milano, \\ Italy \\ ${ }^{b}$ Departament de Ciència de Materials i Química Física and Institut de Química Teórica i Computacional \\ (IQTCUB), Universitat de Barcelona, 08028 Barcelona, Spain.
}

\section{Cartesian coordinates for the optimized structures of $\mathrm{Au}_{1}$ adsorbed on $\mathrm{Zr}_{80} \mathrm{O}_{160}, \mathrm{Zr}_{40} \mathrm{O}_{80}$, and $\mathrm{Zr}_{16} \mathrm{O}_{32}$ nanoparticles.}

\section{$\mathrm{O}_{160} \mathrm{Zr}_{80} \mathrm{Au}_{1}$}

$\left(4 \mathrm{O}_{2 \mathrm{c}}\right.$ hollow, Fig. 2a)

\begin{tabular}{lll}
8.30211 & \multicolumn{3}{c}{6.48508} & 11.6825 \\
8.0924 & 9.55591 & 11.9087 \\
7.94851 & 9.53778 & 16.1852 \\
8.01884 & 12.5 & 11.8707 \\
7.88359 & 12.4999 & 16.1901 \\
8.0924 & 15.4441 & 11.9088 \\
7.94852 & 15.4621 & 16.1853 \\
8.3021 & 18.5149 & 11.6827 \\
11.0434 & 6.44802 & 11.8204 \\
11.0086 & 9.42761 & 7.59756 \\
11.0385 & 9.50425 & 11.8509 \\
11.0132 & 9.51651 & 16.1131 \\
11.0159 & 12.5001 & 7.73129 \\
11.0161 & 12.5 & 11.876 \\
10.9624 & 12.4999 & 16.0776 \\
10.3399 & 12.4999 & 20.0313 \\
11.0086 & 15.5726 & 7.59764 \\
11.0385 & 15.4958 & 11.8509 \\
11.0132 & 15.4834 & 16.1131 \\
11.0434 & 18.552 & 11.8205 \\
13.9566 & 6.44802 & 11.8204 \\
13.9916 & 9.42759 & 7.59761 \\
13.9616 & 9.50424 & 11.8509 \\
13.9867 & 9.51651 & 16.1131 \\
13.9843 & 12.5001 & 7.73135 \\
13.984 & 12.5 & 11.8761 \\
14.0376 & 12.4999 & 16.0776 \\
14.6599 & 12.4999 & 20.0314 \\
13.9916 & 15.5726 & 7.5977 \\
13.9616 & 15.4957 & 11.851 \\
13.9868 & 15.4834 & 16.1132 \\
13.9567 & 18.552 & 11.8206 \\
16.698 & 6.48507 & 11.6827 \\
16.9077 & 9.55589 & 11.9089 \\
17.0514 & 9.53777 & 16.1854 \\
16.9812 & 12.5 & 11.8708 \\
17.1163 & 12.4999 & 16.1903 \\
16.9077 & 15.4441 & 11.9089 \\
17.0514 & 15.4621 & 16.1854 \\
16.698 & 18.5149 & 11.6828 \\
6.49188 & 8.30671 & 14.2337 \\
6.44396 & 11.0383 & 14.0519 \\
& \multicolumn{1}{c}{1} \\
\hline
\end{tabular}

$\begin{array}{lll}6.44396 & 13.9616 & 14.0519 \\ 6.49189 & 16.6932 & 14.2338 \\ 9.53415 & 7.94817 & 9.69515 \\ 9.56122 & 8.10104 & 13.9849 \\ 9.50527 & 11.0055 & 9.75939 \\ 9.50612 & 11.0414 & 14.0146 \\ 9.40109 & 11.0003 & 18.2268 \\ 9.50527 & 13.9946 & 9.75943 \\ 9.50613 & 13.9586 & 14.0146 \\ 9.4011 & 13.9996 & 18.2269 \\ 9.53417 & 17.0519 & 9.69524 \\ 9.56123 & 16.8989 & 13.985 \\ 12.5001 & 7.89706 & 9.68168 \\ 12.5 & 8.02331 & 14.0093 \\ 12.5002 & 10.8733 & 5.61542 \\ 12.5001 & 10.9659 & 9.77795 \\ 12.5 & 11.0198 & 13.9912 \\ 12.4999 & 11.0375 & 18.1814 \\ 12.5002 & 14.127 & 5.61547 \\ 12.5001 & 14.0341 & 9.77799 \\ 12.5 & 13.9801 & 13.9912 \\ 12.4999 & 13.9624 & 18.1815 \\ 12.5001 & 17.103 & 9.68176 \\ 12.5 & 16.9766 & 14.0094 \\ 15.466 & 7.94814 & 9.69522 \\ 15.4388 & 8.10103 & 13.985 \\ 15.4949 & 11.0055 & 9.75949 \\ 15.4939 & 11.0413 & 14.0147 \\ 15.5988 & 11.0002 & 18.2269 \\ 15.4949 & 13.9946 & 9.75952 \\ 15.4939 & 13.9586 & 14.0147 \\ 15.5988 & 13.9996 & 18.227 \\ 15.466 & 17.0519 & 9.6953 \\ 15.4388 & 16.8989 & 13.985 \\ 18.5081 & 8.30668 & 14.2339 \\ 18.556 & 11.0383 & 14.0521 \\ 18.556 & 13.9616 & 14.0521 \\ 18.5081 & 16.6932 & 14.234 \\ 8.30778 & 6.49182 & 14.2403 \\ 7.94976 & 9.53341 & 9.68928 \\ 8.09639 & 9.56114 & 13.9768 \\ 7.8993 & 12.5 & 9.6743 \\ 8.01829 & 12.5 & 13.9987 \\ 7.94976 & 15.4667 & 9.68936 \\ 8.0964 & 15.4388 & 13.9768 \\ 8.30779 & 18.5081 & 14.2404\end{array}$

\begin{tabular}{lll}
11.0397 & \multicolumn{1}{l}{6.45087} & 14.0658 \\
11.0068 & 9.50254 & 9.76242 \\
11.0401 & 9.50723 & 14.0238 \\
10.9882 & 9.4496 & 18.27 \\
10.8739 & 12.5002 & 5.61468 \\
10.9655 & 12.5 & 9.77339 \\
11.0173 & 12.5 & 13.9744 \\
11.0254 & 12.4999 & 18.0971 \\
11.0068 & 15.4975 & 9.76249 \\
11.0401 & 15.4927 & 14.0239 \\
10.9882 & 15.5502 & 18.2701 \\
11.0397 & 18.5491 & 14.0659 \\
13.9603 & 6.45087 & 14.0658 \\
13.9934 & 9.50253 & 9.76247 \\
13.9599 & 9.50723 & 14.0238 \\
14.0116 & 9.4496 & 18.27 \\
14.1264 & 12.5001 & 5.61475 \\
14.0347 & 12.5 & 9.77344 \\
13.9827 & 12.5 & 13.9745 \\
13.9745 & 12.4999 & 18.0972 \\
13.9934 & 15.4975 & 9.76255 \\
13.9599 & 15.4927 & 14.0239 \\
14.0116 & 15.5502 & 18.2701 \\
13.9603 & 18.5491 & 14.0659 \\
16.6922 & 6.4918 & 14.2404 \\
17.0504 & 9.53337 & 9.68944 \\
16.9036 & 9.56113 & 13.9769 \\
17.1009 & 12.5 & 9.67446 \\
16.9817 & 12.5 & 13.9988 \\
17.0504 & 15.4667 & 9.68951 \\
16.9036 & 15.4388 & 13.977 \\
16.6922 & 18.5081 & 14.2406 \\
6.48628 & 8.29563 & 11.675 \\
6.44735 & 11.0408 & 11.8097 \\
6.44735 & 13.9593 & 11.8097 \\
6.48628 & 16.7044 & 11.6751 \\
9.5595 & 8.09152 & 11.9139 \\
9.54082 & 7.9538 & 16.1936 \\
9.42974 & 11.0077 & 7.59547 \\
9.50219 & 11.0367 & 11.8458 \\
9.49973 & 11.0122 & 16.0901 \\
9.42974 & 13.9924 & 7.5955 \\
9.5022 & 13.9633 & 11.8459 \\
9.49973 & 13.9877 & 16.0901 \\
9.5595 & 16.9085 & 11.914 \\
9.54084 & 17.0461 & 16.1936
\end{tabular}

$\begin{array}{lll}11.0397 & 6.45087 & 14.0658\end{array}$

$11.0401 \quad 9.50723 \quad 14.0238$

$\begin{array}{lll}10.9882 & 9.4496 & 18.27\end{array}$

$10.8739 \quad 12.5002 \quad 5.61468$

$10.9655 \quad 12.5 \quad 9.77339$

$11.0173 \quad 12.5 \quad 13.9744$

$\begin{array}{lll}11.0401 & 15.4927 & 14.0239\end{array}$

$\begin{array}{lll}10.9882 & 15.5502 & 18.2701\end{array}$

$\begin{array}{lll}13.9934 & 9.50253 & 9.76247\end{array}$

$\begin{array}{lll}13.9599 & 9.50723 & 14.0238\end{array}$

$\begin{array}{lll}14.0116 & 9.4496 & 18.27\end{array}$

$\begin{array}{lll}14.0347 & 12.5 & 9.77344\end{array}$

$\begin{array}{lll}13.9827 & 12.5 & 13.9745\end{array}$

$\begin{array}{lll}13.9745 & 12.4999 & 18.0972\end{array}$

$\begin{array}{lll}13.9934 & 15.4975 & 9.76255\end{array}$

$\begin{array}{lll}13.9599 & 15.4927 & 14.0239\end{array}$

$\begin{array}{lll}14.0116 & 15.5502 & 18.2701\end{array}$

$\begin{array}{lll}17.0504 & 9.53337 & 9.68944\end{array}$

$\begin{array}{lll}16.9036 & 9.56113 & 13.9769\end{array}$

$\begin{array}{lll}17.1009 & 12.5 & 9.67446\end{array}$

$\begin{array}{lll}17.0504 & 15.4667 & 9.68951\end{array}$

$\begin{array}{lll}16.9036 & 15.4388 & 13.977\end{array}$

$\begin{array}{lll}16.6922 & 18.5081 & 14.2406\end{array}$

$\begin{array}{lll}6.48628 & 8.29563 & 11.675\end{array}$

$\begin{array}{lll}6.44735 & 11.0408 & 11.8097\end{array}$

$\begin{array}{lll}9.5595 & 8.09152 & 11.9139\end{array}$

$\begin{array}{lll}9.54082 & 7.9538 & 16.1936\end{array}$

$\begin{array}{lll}9.42974 & 11.0077 & 7.59547\end{array}$

$\begin{array}{llll}9.50219 & 11.0367 & 11.8458\end{array}$

$\begin{array}{lll}9.49973 & 11.0122 & 16.0901\end{array}$

$\begin{array}{llll}9.42974 & 13.9924 & 7.5955\end{array}$

$\begin{array}{lll}9.5022 & 13.9633 & 11.8459\end{array}$

$\begin{array}{lll}9.5595 & 16.9085 & 11.914\end{array}$

$9.54084 \quad 17.0461 \quad 16.1936$ 


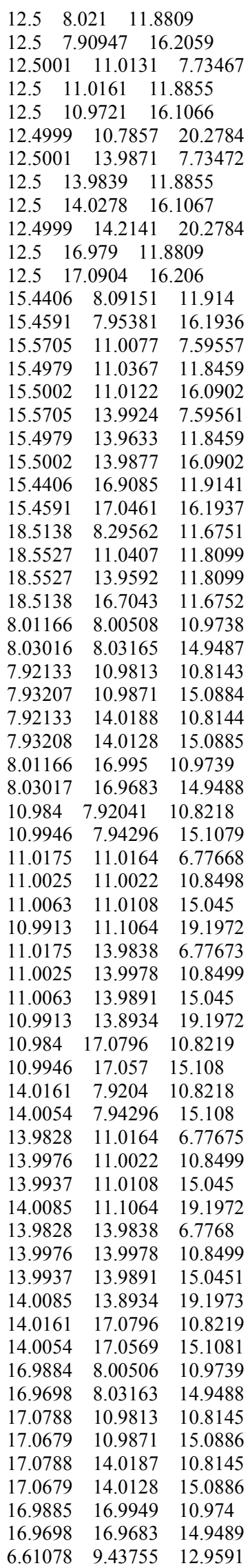

$$
\begin{array}{lll}
6.62692 & 12.5 & 12.944 \\
6.61078 & 15.5624 & 12.9591 \\
9.44328 & 6.61363 & 12.9706 \\
9.52396 & 9.5218 & 8.84287 \\
9.57342 & 9.57282 & 12.9477 \\
9.55334 & 9.56023 & 17.0706 \\
9.41043 & 12.5001 & 8.72456 \\
9.51391 & 12.5 & 12.9431 \\
9.41279 & 12.4999 & 17.1859 \\
9.52396 & 15.4783 & 8.84295 \\
9.57342 & 15.4272 & 12.9477 \\
9.55336 & 15.4396 & 17.0706 \\
9.44328 & 18.3863 & 12.9707 \\
12.5 & 6.6301 & 12.9586 \\
12.5001 & 9.40851 & 8.72789 \\
12.5 & 9.51553 & 12.9521 \\
12.4999 & 9.46853 & 17.2322 \\
12.5002 & 12.5002 & 4.97014 \\
12.5001 & 12.5001 & 8.83792 \\
12.5 & 12.5 & 12.9434 \\
12.4999 & 12.4999 & 17.0722 \\
12.5001 & 15.5916 & 8.72797 \\
12.5 & 15.4844 & 12.9522 \\
12.4999 & 15.5313 & 17.2322 \\
12.5 & 18.3699 & 12.9587 \\
15.5567 & 6.61362 & 12.9706 \\
15.4762 & 9.52178 & 8.84298 \\
15.4266 & 9.57282 & 12.9478 \\
15.4465 & 9.56021 & 17.0706 \\
15.5897 & 12.5 & 8.72468 \\
15.4861 & 12.5 & 12.9432 \\
15.5871 & 12.4999 & 17.186 \\
15.4762 & 15.4783 & 8.84305 \\
15.4266 & 15.4271 & 12.9478 \\
15.4465 & 15.4396 & 17.0707 \\
15.5568 & 18.3863 & 12.9707 \\
18.3892 & 9.43753 & 12.9592 \\
18.3731 & 12.5 & 12.9442 \\
18.3893 & 15.5624 & 12.9593 \\
12.4999 & 12.4998 & 20.6108 \\
\hline \multicolumn{4}{c}{} \\
\hline \multicolumn{4}{c}{}
\end{array}
$$

\section{$\mathrm{O}_{160} \mathrm{Zr}_{80} \mathrm{Au}_{1}$} (Zr top, Fig. 2b)

\begin{tabular}{lll}
8.30717 & \multicolumn{1}{l}{6.48082} & 11.6696 \\
8.09354 & 9.56495 & 11.8978 \\
7.9685 & 9.58761 & 16.1761 \\
8.00647 & 12.5002 & 11.8521 \\
7.80504 & 12.5 & 16.2029 \\
8.09279 & 15.436 & 11.8982 \\
7.96829 & 15.4122 & 16.1767 \\
8.30699 & 18.5204 & 11.67 \\
11.0407 & 6.45599 & 11.8145 \\
11.0056 & 9.42517 & 7.57139 \\
11.0264 & 9.49814 & 11.8158 \\
10.9482 & 9.50567 & 16.0627 \\
11.0258 & 12.4999 & 7.70392 \\
11.0317 & 12.5001 & 11.815 \\
11.1767 & 12.5 & 15.9161 \\
10.741 & 12.5 & 20.1066 \\
11.0056 & 15.5747 & 7.57137 \\
11.026 & 15.5021 & 11.8159 \\
10.9484 & 15.4943 & 16.0627 \\
11.0411 & 18.5442 & 11.8146 \\
13.9594 & 6.45587 & 11.8146 \\
13.9951 & 9.42522 & 7.57147
\end{tabular}

$\begin{array}{lll}8.30717 & 6.48082 & 11.6696\end{array}$ $\begin{array}{lll}8.09354 & 9.56495 & 11.8978\end{array}$ $\begin{array}{lll}13.9739 & 9.49805 & 11.8158\end{array}$ $\begin{array}{lll}14.052 & 9.50554 & 16.0629\end{array}$

$\begin{array}{lll}13.9745 & 12.4999 & 7.70396\end{array}$

$\begin{array}{lll}13.9685 & 12.5 & 11.8152\end{array}$

$\begin{array}{lll}13.8245 & 12.4999 & 15.917\end{array}$

$\begin{array}{lll}14.2585 & 12.4999 & 20.1071\end{array}$

$\begin{array}{lll}13.9953 & 15.5745 & 7.57152\end{array}$

$\begin{array}{lll}13.9737 & 15.5018 & 11.8158\end{array}$

$\begin{array}{lll}14.052 & 15.4943 & 16.0628\end{array}$

$\begin{array}{lll}13.9594 & 18.5439 & 11.8148\end{array}$

$\begin{array}{lll}16.6929 & 6.48051 & 11.6698\end{array}$

$\begin{array}{lll}16.9068 & 9.56456 & 11.8981\end{array}$

$\begin{array}{lll}17.0318 & 9.58728 & 16.1765\end{array}$

$\begin{array}{lll}16.9937 & 12.4999 & 11.8523\end{array}$

$\begin{array}{lll}17.1949 & 12.4998 & 16.2029\end{array}$

$\begin{array}{lll}16.9066 & 15.4353 & 11.8982\end{array}$

$\begin{array}{lll}17.0318 & 15.4123 & 16.1765\end{array}$

$\begin{array}{lll}16.6925 & 18.5194 & 11.6699\end{array}$

$\begin{array}{lll}6.48382 & 8.32215 & 14.2569\end{array}$

$\begin{array}{lll}6.45725 & 11.045 & 14.0526\end{array}$

$\begin{array}{lll}6.45703 & 13.9551 & 14.0529\end{array}$

$\begin{array}{lll}6.48322 & 16.6786 & 14.258\end{array}$

$\begin{array}{lll}9.53175 & 7.95582 & 9.70069\end{array}$

$\begin{array}{lll}9.56621 & 8.10346 & 13.9882\end{array}$

$\begin{array}{llll}9.49413 & 10.9983 & 9.76987\end{array}$

$\begin{array}{lll}9.49509 & 11.0252 & 14.0527\end{array}$

$\begin{array}{lll}9.3153 & 10.974 & 18.3159\end{array}$

$\begin{array}{lll}9.49379 & 14.0016 & 9.77011\end{array}$

$\begin{array}{lll}9.49489 & 13.9751 & 14.0528\end{array}$

$\begin{array}{llll}9.31543 & 14.0259 & 18.3161\end{array}$

$\begin{array}{lll}9.53145 & 17.0441 & 9.7009\end{array}$

$\begin{array}{lll}9.56547 & 16.897 & 13.9885\end{array}$

$\begin{array}{lll}12.5002 & 7.89958 & 9.68309\end{array}$

$\begin{array}{lll}12.5001 & 8.01342 & 14.0211\end{array}$

$\begin{array}{lll}12.5003 & 10.9138 & 5.61681\end{array}$

$\begin{array}{lll}12.5001 & 10.9778 & 9.77766\end{array}$

$\begin{array}{lll}12.5001 & 11.0436 & 13.9953\end{array}$

$\begin{array}{lll}12.4999 & 11.1172 & 18.1368\end{array}$

$\begin{array}{lll}12.5003 & 14.086 & 5.6169\end{array}$

$\begin{array}{lll}12.5 & 14.022 & 9.77784\end{array}$

$\begin{array}{lll}12.4999 & 13.9562 & 13.9954\end{array}$

$\begin{array}{lll}12.4998 & 13.8825 & 18.1369\end{array}$

$\begin{array}{lll}12.5001 & 17.1003 & 9.68326\end{array}$

$\begin{array}{lll}12.4998 & 16.9863 & 14.0213\end{array}$

$\begin{array}{lll}15.4687 & 7.95582 & 9.70084\end{array}$

$\begin{array}{lll}15.434 & 8.10328 & 13.9883\end{array}$

$\begin{array}{lll}15.5062 & 10.9983 & 9.76993\end{array}$

$\begin{array}{lll}15.505 & 11.0251 & 14.0527\end{array}$

$\begin{array}{lll}15.6846 & 10.974 & 18.3159\end{array}$

$\begin{array}{lll}15.5061 & 14.0015 & 9.76998\end{array}$

$\begin{array}{lll}15.5049 & 13.9747 & 14.0528\end{array}$

$\begin{array}{lll}15.6847 & 14.0257 & 18.3159\end{array}$

$\begin{array}{lll}15.4687 & 17.0438 & 9.70106\end{array}$

$\begin{array}{lll}15.4336 & 16.8964 & 13.9885\end{array}$

$\begin{array}{lll}18.5162 & 8.32178 & 14.2572\end{array}$

$\begin{array}{lll}18.543 & 11.0448 & 14.0529\end{array}$

$\begin{array}{lll}18.5429 & 13.955 & 14.0529\end{array}$

$\begin{array}{lll}18.516 & 16.6782 & 14.2573\end{array}$

$\begin{array}{lll}8.2972 & 6.50563 & 14.2243\end{array}$

$\begin{array}{lll}7.94091 & 9.52805 & 9.68136\end{array}$

$\begin{array}{llll}8.09503 & 9.55479 & 13.9708\end{array}$

$\begin{array}{lll}7.89675 & 12.4997 & 9.66084\end{array}$

$\begin{array}{lll}8.03185 & 12.5001 & 13.9844\end{array}$

$\begin{array}{lll}7.94033 & 15.4715 & 9.68113\end{array}$

$\begin{array}{lll}8.09452 & 15.446 & 13.971\end{array}$

$\begin{array}{lll}8.29708 & 18.4953 & 14.2253\end{array}$

$\begin{array}{lll}11.0291 & 6.44593 & 14.0547\end{array}$ 


\begin{tabular}{|c|c|c|}
\hline & & \\
\hline S.0J5. & & \\
\hline (005. & & \\
\hline & & \\
\hline & \multicolumn{2}{|c|}{$12.5 \quad 9.73312$} \\
\hline & 12.4999 & \\
\hline & & \\
\hline & & \\
\hline & & \\
\hline & 15. & \\
\hline & & \\
\hline & 6.4 & \\
\hline & & \\
\hline & & \\
\hline & & \\
\hline & & \\
\hline & & \\
\hline & 12. & \\
\hline & & \\
\hline & & \\
\hline & & \\
\hline & & \\
\hline & & \\
\hline & & \\
\hline & & \\
\hline & & \\
\hline & & \\
\hline & & \\
\hline & & \\
\hline & & \\
\hline & & \\
\hline & & \\
\hline & & \\
\hline & & \\
\hline & & \\
\hline & & \\
\hline & & \\
\hline & & \\
\hline & & \\
\hline & & \\
\hline & & \\
\hline & & \\
\hline & & \\
\hline & & \\
\hline & & \\
\hline & & \\
\hline 2.57 . & $.93131 \quad 16$ & \\
\hline 12.5002 & 11.0046 & \\
\hline & & \\
\hline & & \\
\hline & & \\
\hline .5002 & 13.9954 & \\
\hline $2.5 \quad 12$ & $4.0025 \quad 11$ & \\
\hline .5002 & 14.1474 & \\
\hline & & \\
\hline & & \\
\hline & & \\
\hline & & \\
\hline & & \\
\hline & & \\
\hline & & \\
\hline & & \\
\hline & & \\
\hline & & \\
\hline & & \\
\hline & & \\
\hline .498 & 17.0451 & \\
\hline
\end{tabular}

\begin{tabular}{|c|c|c|}
\hline 18.5045 & 8.28898 & 11.695 \\
\hline 18.5598 & 11.0354 & 11.8122 \\
\hline 18.5597 & 13.9645 & 11.8122 \\
\hline 18.5043 & 16.7111 & 11.6951 \\
\hline 8.01613 & 8.01117 & 10.9705 \\
\hline 8.04271 & 8.04035 & 14.9575 \\
\hline 7.91402 & 10.9829 & 10.8106 \\
\hline 7.94967 & 11.0139 & 15.1005 \\
\hline 7.91355 & 14.0178 & 10.8109 \\
\hline 7.94948 & 13.9864 & 15.1006 \\
\hline 8.01543 & 16.9902 & 10.9714 \\
\hline 8.04218 & 16.9604 & 14.9579 \\
\hline 10.9856 & 7.92805 & 10.8166 \\
\hline 10.9785 & 7.95253 & 15.1047 \\
\hline 11.0197 & 11.0145 & 6.76373 \\
\hline 11.0023 & 11.0028 & 10.8365 \\
\hline 11.0167 & 11.0166 & 15.0403 \\
\hline 11.0849 & 11.0833 & 19.2073 \\
\hline 11.0198 & 13.9853 & 6.76382 \\
\hline 11.0022 & 13.9972 & 10.8366 \\
\hline 11.0167 & 13.9832 & 15.0403 \\
\hline 11.0849 & 13.9166 & 19.2073 \\
\hline 10.9853 & 17.0722 & 10.817 \\
\hline 10.9783 & 17.0475 & 15.1049 \\
\hline 14.0147 & 7.92796 & 10.8167 \\
\hline 14.0215 & 7.95246 & 15.1047 \\
\hline 13.9807 & 11.0145 & 6.76372 \\
\hline 13.998 & 11.0027 & 10.8365 \\
\hline 13.9835 & 11.0165 & 15.0404 \\
\hline 13.9146 & 11.0832 & 19.2077 \\
\hline 13.9807 & 13.9853 & 6.76383 \\
\hline 13.998 & 13.9971 & 10.8366 \\
\hline 13.9834 & 13.9831 & 15.0404 \\
\hline 13.9145 & 13.9167 & 19.2078 \\
\hline 14.0144 & 17.0718 & 10.8168 \\
\hline 14.0212 & 17.0473 & 15.1048 \\
\hline 16.9841 & 8.01067 & 10.9709 \\
\hline 16.9574 & 8.03996 & 14.9576 \\
\hline 17.0862 & 10.9825 & 10.8109 \\
\hline 17.0505 & 11.0136 & 15.1007 \\
\hline 17.0862 & 14.0174 & 10.8109 \\
\hline 17.0504 & 13.9861 & 15.1007 \\
\hline 16.9837 & 16.9892 & 10.9709 \\
\hline 16.957 & 16.9598 & 14.9578 \\
\hline 6.61582 & 9.44846 & 12.9734 \\
\hline 6.62782 & 12.5003 & 12.9426 \\
\hline 6.61513 & 15.5528 & 12.9741 \\
\hline 9.45166 & 6.61702 & 12.9627 \\
\hline 9.51767 & 9.52581 & 8.83499 \\
\hline 9.57464 & 9.57909 & 12.9472 \\
\hline 9.54386 & 9.60841 & 17.0817 \\
\hline 9.40967 & 12.5 & .71904 \\
\hline 9.51515 & $12.5 \quad 12$ & 2.9377 \\
\hline 9.26052 & 12.5001 & 17.369 \\
\hline 9.51725 & 15.4741 & 8.83533 \\
\hline 9.57444 & 15.4211 & 12.9474 \\
\hline 9.54402 & 15.3917 & 17.0817 \\
\hline 9.45101 & 18.3837 & 12.9635 \\
\hline 12.5001 & 6.63278 & 12.9636 \\
\hline 12.5002 & 9.40619 & 8.71053 \\
\hline 12.5001 & 9.51675 & 12.9404 \\
\hline 12.5001 & 9.4564 & 17.1962 \\
\hline 12.5002 & 12.4999 & 4.86753 \\
\hline 12.5001 & 12.4999 & 8.8259 \\
\hline 12.5001 & 12.4999 & 12.9245 \\
\hline 12.5002 & 12.4999 & 17.0262 \\
\hline $2.5 \quad 1$ & 5937 & 1072 \\
\hline
\end{tabular}

\begin{tabular}{lll}
12.5 & 15.483 & 12.9405 \\
12.5001 & 15.5434 & 17.1961 \\
12.4998 & 18.367 & 12.9637 \\
15.5486 & 6.6168 & 12.963 \\
15.4827 & 9.52579 & 8.83509 \\
15.4256 & 9.57892 & 12.9473 \\
15.4563 & 9.60817 & 17.0819 \\
15.5906 & 12.4999 & 8.71902 \\
15.4851 & 12.4999 & 12.9378 \\
15.7388 & 12.4999 & 17.3688 \\
15.4827 & 15.474 & 8.83515 \\
15.4255 & 15.4208 & 12.9474 \\
15.4564 & 15.3916 & 17.0819 \\
15.548 & 18.3829 & 12.9631 \\
18.3844 & 9.44783 & 12.9737 \\
18.3723 & 12.4999 & 12.9429 \\
18.3843 & 15.5519 & 12.9738 \\
12.5003 & 12.5 & 2.67809 \\
\hline \multicolumn{4}{c}{} \\
\hline
\end{tabular}

$\mathrm{O}_{160} \mathrm{Zr}_{80} \mathrm{Au}_{1}$ (ZrO bridge, Fig. 2c)

$\begin{array}{lcc}8.31152 & 6.47465 & 11.6938 \\ 8.10521 & 9.55489 & 11.9094 \\ 8.00293 & 9.59654 & 16.1888 \\ 8.01044 & 12.4973 & 11.8559 \\ 7.80215 & 12.5002 & 16.2096 \\ 8.09629 & 15.4261 & 11.895 \\ 7.9817 & 15.3879 & 16.1687 \\ 8.30276 & 18.5175 & 11.6666 \\ 11.0563 & 6.44371 & 11.7982 \\ 11.011 & 9.4214 & 7.57499 \\ 11.0359 & 9.50375 & 11.8269 \\ 10.9922 & 9.53264 & 16.0745 \\ 11.023 & 12.4963 & 7.69894 \\ 11.0471 & 12.4922 & 11.8158 \\ 11.2993 & 12.4782 & 15.8724 \\ 10.6777 & 12.547 & 20.0786 \\ 10.9986 & 15.5725 & 7.56417 \\ 11.0294 & 15.5023 & 11.8113 \\ 10.958 & 15.4899 & 16.0501 \\ 11.0382 & 18.5434 & 11.8008 \\ 13.9563 & 6.4562 & 11.7608 \\ 14.0015 & 9.42819 & 7.57422 \\ 13.9867 & 9.49325 & 11.8345 \\ 14.2273 & 9.39019 & 16.1235 \\ 13.9783 & 12.5033 & 7.70946 \\ 13.9787 & 12.508 & 11.8512 \\ 13.9868 & 12.5239 & 16.0434 \\ 14.209 & 12.5886 & 20.1907 \\ 13.9909 & 15.5777 & 7.56767 \\ 13.9763 & 15.5034 & 11.8187 \\ 14.0327 & 15.5321 & 16.0673 \\ 13.9459 & 18.5547 & 11.7974 \\ 16.6744 & 6.47228 & 11.582 \\ 16.9113 & 9.57203 & 11.8862 \\ 16.9363 & 9.48672 & 16.1617 \\ 16.9941 & 12.5004 & 11.8613 \\ 17.1556 & 12.5334 & 16.179 \\ 16.9045 & 15.4512 & 11.8948 \\ 17.0424 & 15.4842 & 16.173 \\ 16.689 & 18.5339 & 11.6621 \\ 6.50089 & 8.32124 & 14.2789 \\ 6.46832 & 11.044 & 14.0685 \\ 6.4711 & 13.9377 & 14.0606 \\ 6.48191 & 16.6641 & 14.2656\end{array}$


Supporting information

\begin{tabular}{|c|c|c|}
\hline .53378 & 7.94936 & 9.70781 \\
\hline 9.57093 & 8.10689 & 13.9989 \\
\hline 9.49991 & 10.9937 & 9.77838 \\
\hline 9.5291 & 11.015 & 14.0681 \\
\hline 9.31992 & 10.9743 & 18.335 \\
\hline 9.48843 & 13.9956 & 9.77604 \\
\hline 9.50281 & 13.9724 & 14.0613 \\
\hline 9.27233 & 14.0547 & 18.3133 \\
\hline $9.524 \quad 1$ & 17.0376 & 9.69697 \\
\hline 9.57071 & 16.8908 & 13.9837 \\
\hline 12.5123 & 7.90312 & 9.67841 \\
\hline 12.5247 & 7.99467 & 14.0128 \\
\hline 12.5029 & 10.9038 & 5.60422 \\
\hline 12.4993 & 10.9795 & 9.78454 \\
\hline 12.5217 & 11.0348 & 14.0164 \\
\hline 12.4613 & 11.1457 & 18.1645 \\
\hline 12.4967 & 14.0896 & 5.59905 \\
\hline 12.5029 & 14.023 & 9.777 \\
\hline 12.5267 & 13.9539 & 13.9954 \\
\hline 12.483 & 13.929 & 18.139 \\
\hline 12.4919 & 17.1008 & 9.67401 \\
\hline 12.4953 & 16.9911 & 14.0095 \\
\hline 15.4704 & 7.97016 & 9.68834 \\
\hline 15.4471 & 8.09571 & 14.0003 \\
\hline 15.5129 & 11.0091 & 9.77999 \\
\hline 15.5384 & 11.0111 & 14.0637 \\
\hline 15.7029 & 11.1008 & 18.3303 \\
\hline 15.4996 & 14.0053 & 9.76711 \\
\hline 15.5015 & 13.9778 & 14.0334 \\
\hline 15.6119 & 14.089 & 18.2755 \\
\hline 15.4643 & 17.0529 & 9.69104 \\
\hline 15.4323 & 16.9108 & 13.984 \\
\hline 18.5761 & 8.32738 & 14.2298 \\
\hline 18.5446 & 11.0577 & 14.0662 \\
\hline 18.5505 & 13.9712 & 14.0501 \\
\hline 18.5134 & 16.7051 & 14.2384 \\
\hline 8.31466 & 6.50413 & 14.2529 \\
\hline 7.94234 & 9.52599 & 9.68858 \\
\hline 8.10755 & 9.56168 & 13.9804 \\
\hline $\begin{array}{ll}7.895 & 1\end{array}$ & 12.4875 & 9.66339 \\
\hline 8.05101 & 12.4838 & 13.987 \\
\hline 7.93007 & 15.4602 & 9.67623 \\
\hline 8.09914 & 15.4397 & 13.9649 \\
\hline 8.29396 & 18.487 & 14.2216 \\
\hline 11.0405 & 6.45459 & 14.0464 \\
\hline 11.0132 & 9.50118 & 9.73729 \\
\hline 11.0739 & 9.48852 & 13.9911 \\
\hline 11.0252 & 9.46961 & 18.2153 \\
\hline 10.9018 & 12.4924 & 5.58291 \\
\hline 10.9498 & 12.5002 & 9.73205 \\
\hline 10.9944 & 12.5034 & 13.8776 \\
\hline 10.901 & 12.543 & 17.9749 \\
\hline 11.0048 & 15.4853 & 9.72575 \\
\hline 11.0617 & 15.4772 & 13.975 \\
\hline 10.9765 & 15.5584 & 18.1984 \\
\hline 11.0244 & 18.5584 & 14.0437 \\
\hline 13.983 & 6.41914 & 13.9902 \\
\hline 13.9949 & 9.52392 & 9.73851 \\
\hline 13.9577 & 9.51011 & 14.0161 \\
\hline 13.9987 & 9.58338 & 18.2886 \\
\hline 14.0952 & 12.4989 & 5.59048 \\
\hline 14.0458 & 12.4975 & 9.75157 \\
\hline 14.0229 & 12.4718 & 13.957 \\
\hline 14.0247 & 12.5372 & 18.1197 \\
\hline 13.9871 & 15.4962 & 9.73248 \\
\hline 13.9518 & 15.501 & 13.9884 \\
\hline 13.9646 & 15.6049 & 18.2173 \\
\hline
\end{tabular}

\begin{tabular}{|c|c|c|c|c|c|}
\hline 13.9598 & 18.57 & 14.0397 & 10.9783 & 17.0723 & 10.818 \\
\hline 16.7976 & 6.51198 & 14.073 & 10.9771 & 17.0528 & 15.1011 \\
\hline 17.0681 & 9.54179 & 9.66915 & 14.0206 & 7.92962 & 10.8092 \\
\hline 16.9419 & 9.53795 & 13.9524 & 14.0075 & 7.9115 & 15.0721 \\
\hline 17.1058 & 12.5104 & 9.66451 & 13.9942 & 11.0062 & 6.78715 \\
\hline 16.973 & 12.5089 & 13.9931 & 14.0052 & 11.0047 & 10.8564 \\
\hline 17.0552 & 15.473 & 9.67622 & 14.036 & 10.9885 & 15.0845 \\
\hline 16.905 & 15.4597 & 13.9666 & 13.8316 & 11.1694 & 19.3211 \\
\hline 16.6941 & 18.5199 & 14.2173 & 13.9909 & 13.9993 & 6.78499 \\
\hline 6.50016 & 8.28378 & 11.7129 & 13.9991 & 14.0018 & 10.8476 \\
\hline 6.44734 & 11.0268 & 11.8237 & 13.9959 & 14.0016 & 15.0501 \\
\hline 6.43712 & 13.9528 & 11.8178 & 13.8698 & 13.9832 & 19.2574 \\
\hline 6.49277 & 16.7038 & 11.7021 & 14.0138 & 17.0844 & 10.8191 \\
\hline 9.56753 & 8.08273 & 11.9315 & 14.0144 & 17.0761 & 15.0968 \\
\hline 9.54427 & 7.97268 & 16.2032 & 16.9958 & 8.01207 & 10.95 \\
\hline 9.43107 & 11.0026 & 7.60339 & 17.033 & 7.99805 & 14.8865 \\
\hline 9.51071 & 11.0431 & 11.8742 & 17.0906 & 10.9918 & 10.8209 \\
\hline 9.56799 & 11.156 & 16.1844 & 17.0536 & 11.0084 & 15.0999 \\
\hline 9.42644 & 13.9812 & 7.60023 & 17.0869 & 14.0279 & 10.8205 \\
\hline 9.51774 & 13.9463 & 11.8714 & 17.0639 & 14.0206 & 15.1066 \\
\hline 9.57448 & 13.8408 & 16.1745 & 16.9875 & 17.0111 & 10.972 \\
\hline 9.54862 & 16.9054 & 11.919 & 16.965 & 16.9835 & 14.947 \\
\hline 9.50042 & 17.0392 & 16.1876 & 6.62421 & 9.43821 & 12.9919 \\
\hline 12.5034 & 8.02969 & 11.8825 & 6.6356 & 12.4898 & 12.9549 \\
\hline 12.5179 & 7.94888 & 16.2032 & 6.61457 & 15.5518 & 12.9797 \\
\hline 12.5025 & 11.0049 & 7.72858 & 9.45207 & 6.61218 & 12.9865 \\
\hline 12.514 & 10.9952 & 11.899 & 9.51004 & 9.51433 & 8.85453 \\
\hline 12.6153 & 10.8272 & 16.1409 & 9.58445 & 9.57378 & 12.9561 \\
\hline 12.4312 & 10.7202 & 20.1672 & 9.58096 & 9.6257 & 17.09 \\
\hline 12.4967 & 13.9949 & 7.72433 & 9.40359 & 12.4954 & 8.73633 \\
\hline 12.501 & 13.9981 & 11.8903 & 9.52514 & 12.4974 & 12.9419 \\
\hline 12.5292 & 14.1466 & 16.1151 & 9.2276 & 12.5195 & 17.4008 \\
\hline 12.4449 & 14.4197 & 20.1167 & 9.50006 & 15.4734 & 8.84703 \\
\hline 12.4996 & 16.9753 & 11.8775 & 9.57751 & 15.4178 & 12.9488 \\
\hline 12.4812 & 17.0985 & 16.1931 & 9.54858 & 15.3896 & 17.0714 \\
\hline 15.4645 & 8.09399 & 11.9191 & 9.44111 & 18.3816 & 12.9619 \\
\hline 15.5273 & 7.42061 & 16.0012 & 12.5057 & 6.62122 & 12.933 \\
\hline 15.5725 & 11.0182 & 7.60207 & 12.5031 & 9.40785 & 8.72869 \\
\hline 15.4912 & 11.0463 & 11.8802 & 12.5164 & 9.51667 & 12.9511 \\
\hline 15.5088 & 11.1261 & 16.1751 & 12.5356 & 9.46328 & 17.2361 \\
\hline 15.5688 & 13.9923 & 7.59521 & 12.4981 & 12.4962 & 4.93765 \\
\hline 15.4998 & 13.9568 & 11.8609 & 12.4994 & 12.5005 & 8.83345 \\
\hline 15.4961 & 13.9963 & 16.1256 & 12.5133 & 12.4938 & 12.9462 \\
\hline 15.4429 & 16.9221 & 11.9153 & 12.5265 & 12.5236 & 17.0626 \\
\hline 15.4745 & 17.0986 & 16.1895 & 12.4956 & 15.5974 & 8.72378 \\
\hline 18.5124 & 8.29492 & 11.6744 & 12.5048 & 15.4858 & 12.9406 \\
\hline $18.57 \quad 1$ & 11.0412 & 11.8182 & 12.4964 & 15.5719 & 17.196 \\
\hline 18.5557 & 13.9698 & 11.81 & 12.4919 & 18.373 & 12.9519 \\
\hline 18.5066 & 16.7229 & 11.6801 & 15.5698 & 6.60851 & 12.9111 \\
\hline 8.01381 & 7.9944 & 10.9955 & 15.4945 & 9.5344 & 8.84412 \\
\hline 8.05293 & 8.04341 & 14.9703 & 15.4445 & 9.56842 & 12.9585 \\
\hline 7.91504 & 10.9741 & 10.8263 & 15.5139 & 9.75599 & 17.263 \\
\hline 7.97045 & 11.014 & 15.1112 & 15.5946 & 12.5087 & 8.73814 \\
\hline 7.90542 & 14.0134 & 10.8213 & 15.4887 & 12.5068 & 12.9549 \\
\hline 7.96064 & 13.9771 & 15.0971 & 15.5933 & 12.5947 & 17.2804 \\
\hline 8.00511 & 16.9921 & 10.9796 & 15.4869 & 15.4875 & 8.84486 \\
\hline 8.04036 & 16.9568 & 14.9499 & 15.4288 & 15.4349 & 12.9467 \\
\hline 10.9863 & 7.92254 & 10.8262 & 15.4415 & 15.4768 & 17.0821 \\
\hline 10.9917 & 7.96235 & 15.1097 & 15.553 & 18.4009 & 12.9606 \\
\hline 11.0074 & 10.9983 & 6.78653 & 18.4085 & 9.45535 & 12.9423 \\
\hline 11.0065 & 11.0005 & 10.8471 & 18.3742 & 12.5144 & 12.9565 \\
\hline 11.0447 & 11.0086 & 15.0447 & 18.3866 & 15.5788 & 12.9664 \\
\hline 11.0409 & 11.1299 & 19.2146 & 15.7081 & 7.36602 & 17.6834 \\
\hline 11.0037 & 13.9958 & 6.78338 & & & \\
\hline 11.0045 & 13.9949 & 10.8437 & \multirow{3}{*}{\multicolumn{3}{|c|}{$\begin{array}{l}\mathbf{O}_{\mathbf{8 0}} \mathbf{Z r}_{\mathbf{4 0}} \mathbf{A} \mathbf{u}_{\mathbf{1}} \\
\text { (Zr top, Fig. } 2 \text { d) }\end{array}$}} \\
\hline 11.0347 & 13.9771 & 15.0428 & & & \\
\hline 11.0614 & 13.9635 & 19.2064 & & & \\
\hline
\end{tabular}

$\begin{array}{lll}13.9598 & 18.57 & 14.0397\end{array}$

$\begin{array}{lll}16.7976 & 6.51198 & 14.073\end{array}$

$\begin{array}{lll}17.0681 & 9.54179 & 9.66915\end{array}$

$\begin{array}{lll}16.973 & 12.5089 & 13.9931\end{array}$

$\begin{array}{lll}17.0552 & 15.473 & 9.67622\end{array}$

$\begin{array}{lll}17.0505 & 15.4597 & 13.9666\end{array}$

$\begin{array}{lll}6.49277 & 16.7038 & 11.7021\end{array}$

$\begin{array}{lll}9.56753 & 8.08273 & 11.9315\end{array}$

$\begin{array}{lll}9.54427 & 7.97268 & 16.2032\end{array}$

$\begin{array}{llll}9.51071 & 11.0431 & 11.8742\end{array}$

$\begin{array}{llll}9.56799 & 11.156 & 16.1844\end{array}$

$\begin{array}{lll}9.42644 & 13.9812 & 7.60023\end{array}$

$\begin{array}{lll}9.51774 & 13.9463 & 11.8714\end{array}$

$\begin{array}{lll}9.57448 & 13.8408 & 16.1745\end{array}$

$\begin{array}{llll}12.5025 & 11.0049 & 7.72858\end{array}$

$\begin{array}{lll}12.514 & 10.9952 & 11.899\end{array}$

$\begin{array}{lll}12.6153 & 10.8272 & 16.1409\end{array}$

$\begin{array}{lll}12.501 & 13.9981 & 11.8903\end{array}$

$\begin{array}{lll}12.5292 & 14.1466 & 16.1151\end{array}$

$\begin{array}{lll}12.4449 & 14.4197 & 20.1167\end{array}$

$\begin{array}{lll}12.4996 & 16.9753 & 11.8775\end{array}$

$12.4812 \quad 17.0985 \quad 16.193$

$\begin{array}{lll}15.4645 & 8.09399 & 11.9191\end{array}$

$\begin{array}{lll}15.5273 & 7.42061 & 16.0012\end{array}$

$\begin{array}{lll}15.5725 & 11.0182 & 7.60207\end{array}$

$\begin{array}{lll}15.4912 & 11.0463 & 11.8802\end{array}$

$\begin{array}{lll}15.4998 & 13.9568 & 11.8609\end{array}$

$\begin{array}{lll}15.4961 & 13.9963 & 16.1256\end{array}$

$\begin{array}{lll}15.4429 & 16.9221 & 11.9153\end{array}$

$\begin{array}{lll}15.4745 & 17.0986 & 16.1895\end{array}$

$\begin{array}{lll}18.5124 & 8.29492 & 11.6744\end{array}$

8.05293

$\begin{array}{lll}7.91504 & 10.9741 & 10.8263\end{array}$

$\begin{array}{lll}7.97045 & 11.014 & 15.1112\end{array}$

$\begin{array}{lll}7.90542 & 14.0134 & 10.8213\end{array}$

$\begin{array}{lll}7.96064 & 13.9771 & 15.0971\end{array}$

$\begin{array}{lll}8.00511 & 16.9921 & 10.9796\end{array}$

$\begin{array}{lll}8.04036 & 16.9568 & 14.9499\end{array}$

$\begin{array}{lll}10.9863 & 7.92254 & 10.8262\end{array}$

$\begin{array}{lll}10.9917 & 7.96235 & 15.1097\end{array}$

$\begin{array}{lll}11.0074 & 10.9983 & 6.78653\end{array}$

$\begin{array}{lll}11.0065 & 11.0005 & 10.8471\end{array}$

$11.0447 \quad 11.0086 \quad 15.0447$

$\begin{array}{lll}11.0409 & 11.1299 & 19.2146\end{array}$

$\begin{array}{lll}11.0037 & 13.9958 & 6.78338\end{array}$

$\begin{array}{lll}11.0347 & 13.9771 & 15.0428\end{array}$

$\begin{array}{lll}11.0614 & 13.9635 & 19.2064\end{array}$ 


$\begin{array}{lll}17.1795 & 7.86411 & 14.5108 \\ 18.1551 & 12.6243 & 13.9519 \\ 17.8761 & 15.8491 & 13.9861 \\ 7.17397 & 9.10403 & 13.9674 \\ 6.79244 & 12.2921 & 13.9491 \\ 7.73213 & 17.0949 & 14.6054 \\ 10.6237 & 8.66718 & 8.46338 \\ 10.6145 & 9.01383 & 13.6277 \\ 10.8528 & 12.4768 & 8.70758 \\ 10.9535 & 12.592 & 13.5286 \\ 10.4984 & 12.4278 & 18.667 \\ 10.7749 & 16.274 & 8.31676 \\ 10.0321 & 16.3163 & 13.4534 \\ 14.2381 & 8.70726 & 8.41385 \\ 14.473 & 8.83737 & 13.6952 \\ 14.0977 & 12.4968 & 8.70185 \\ 13.9642 & 12.5137 & 13.545 \\ 14.5641 & 12.5378 & 18.6693 \\ 14.4176 & 16.3028 & 8.45458 \\ 14.37 & 15.9939 & 13.6802 \\ 9.15089 & 7.12393 & 11.0139 \\ 9.00609 & 10.6301 & 11.3199 \\ 8.69722 & 10.5825 & 16.5454 \\ 8.68368 & 14.9678 & 11.5466 \\ 8.72599 & 14.2251 & 16.6833 \\ 7.90509 & 17.2679 & 10.3946 \\ 12.3757 & 6.84488 & 11.0481 \\ 12.4622 & 10.4359 & 6.33076 \\ 12.4863 & 11.0359 & 11.455 \\ 12.5032 & 10.9023 & 16.2982 \\ 12.5722 & 14.5016 & 6.33301 \\ 12.408 & 14.0465 & 11.4714 \\ 12.5232 & 14.1472 & 16.2924 \\ 12.7079 & 18.2075 & 11.0509 \\ 17.1359 & 7.82051 & 10.4892 \\ 16.1626 & 10.527 & 11.3048 \\ 16.2928 & 10.7619 & 16.5862 \\ 15.9862 & 14.3855 & 11.3723 \\ 16.3328 & 14.3763 & 16.5366 \\ 15.896 & 17.826 & 11.0327 \\ 18.5795 & 9.26436 & 12.5306 \\ 17.9085 & 12.5772 & 11.1462 \\ 17.9919 & 15.7017 & 10.8817 \\ 7.03372 & 9.2648 & 10.8895 \\ 7.12773 & 12.4895 & 11.1126 \\ 6.32949 & 15.693 & 12.6204 \\ 10.896 & 9.02099 & 11.1102 \\ 10.7623 & 8.8537 & 16.3071 \\ 10.245 & 12.4758 & 6.34777 \\ 10.2897 & 12.7521 & 11.1155 \\ 10.5636 & 12.4574 & 16.0979 \\ 10.915 & 16.224 & 10.9872 \\ 10.8007 & 16.1623 & 16.3063 \\ 14.1044 & 8.88668 & 11.0799 \\ 14.2093 & 8.87812 & 16.3797 \\ 14.7894 & 12.4993 & 6.353 \\ 14.5046 & 12.4521 & 11.1166 \\ 14.4205 & 12.5453 & 16.0942 \\ 14.0973 & 15.991 & 11.1001 \\ 14.33 & 16.1525 & 16.376 \\ 9.29832 & 7.00812 & 14.1183 \\ 8.84753 & 10.67 & 8.62407 \\ 9.00894 & 10.9028 & 13.8999 \\ 8.83767 & 14.1993 & 8.69371 \\ 8.77597 & 14.085 & 14.0128 \\ 9.30698 & 18.6705 & 12.3796\end{array}$

\begin{tabular}{lll}
12.4228 & 7.09147 & 13.8538 \\
12.4547 & 10.5795 & 8.90583 \\
12.5479 & 10.4954 & 13.8834 \\
12.5007 & 10.2106 & 18.647 \\
12.5426 & 14.4364 & 8.90217 \\
12.2479 & 14.7103 & 13.8845 \\
12.5242 & 14.755 & 18.6522 \\
12.5105 & 17.8723 & 13.8875 \\
15.7357 & 6.42055 & 12.4694 \\
16.1219 & 10.7908 & 8.62033 \\
16.1133 & 10.8956 & 13.9201 \\
16.1463 & 14.2376 & 8.69294 \\
15.979 & 14.104 & 13.8898 \\
15.7352 & 17.9663 & 14.1106 \\
17.5899 & 7.4101 & 12.5 \\
17.7891 & 11.0627 & 12.5836 \\
17.7628 & 14.4646 & 12.5144 \\
7.24755 & 10.4955 & 12.523 \\
7.12441 & 13.8967 & 12.6316 \\
7.94903 & 17.051 & 12.5 \\
10.5354 & 7.23722 & 12.4857 \\
10.7821 & 10.7677 & 7.44173 \\
10.7411 & 10.8089 & 12.5114 \\
10.8171 & 10.733 & 17.5491 \\
10.8918 & 14.1396 & 7.36864 \\
10.6245 & 14.3755 & 12.5 \\
10.8604 & 14.1082 & 17.6314 \\
11.1033 & 17.8756 & 12.3684 \\
13.9373 & 7.21089 & 12.4164 \\
14.139 & 10.8406 & 7.38923 \\
14.2661 & 10.7339 & 12.5 \\
14.1594 & 10.861 & 17.6108 \\
14.267 & 14.1829 & 7.45094 \\
14.1911 & 14.2589 & 12.4886 \\
14.2323 & 14.2179 & 17.5583 \\
14.5045 & 17.7524 & 12.477 \\
8.92981 & 8.91612 & 10.0612 \\
8.97181 & 8.88749 & 14.9395 \\
8.78518 & 12.3664 & 9.77892 \\
8.5522 & 12.3199 & 15.4192 \\
9.20703 & 15.9789 & 9.68624 \\
9.02109 & 15.793 & 15.3138 \\
12.3487 & 8.56649 & 9.61181 \\
12.4655 & 8.83862 & 15.1141 \\
12.4738 & 12.5418 & 10.0604 \\
12.4582 & 12.5262 & 14.9396 \\
12.6801 & 16.4478 & 9.58083 \\
12.6336 & 16.2148 & 15.2211 \\
15.8037 & 9.07704 & 9.77631 \\
15.923 & 9.19631 & 15.2237 \\
16.1614 & 12.5345 & 9.88591 \\
16.4335 & 12.6513 & 15.3882 \\
16.1125 & 16.0282 & 10.0605 \\
16.0839 & 16.0702 & 14.9388 \\
19.4846 & 5.51543 & 12.5 \\
\hline & \multicolumn{3}{l}{} & \\
\hline & &
\end{tabular}

\section{$\mathbf{O}_{80} \mathbf{Z r}_{40} \mathbf{A u}_{1}$}

$\left(\mathrm{O}_{2 \mathrm{c}}\right.$ top, Fig. 2e)

$\begin{array}{lll}17.1892 & 7.7103 & 14.7995 \\ 18.1233 & 12.7515 & 13.7801 \\ 17.9488 & 15.9928 & 13.9078 \\ 7.19874 & 9.19601 & 14.023 \\ 6.82338 & 12.4049 & 14.0456 \\ 7.90129 & 17.2259 & 14.7981 \\ 10.5907 & 8.74579 & 8.46887\end{array}$

\begin{tabular}{|c|c|c|}
\hline 10.8814 & 9.23659 & 13.7716 \\
\hline 10.8933 & 12.5212 & 8.76531 \\
\hline 11.3206 & 12.5704 & 13.6058 \\
\hline 10.3632 & 12.5494 & 18.5848 \\
\hline 10.7346 & 16.3028 & 8.38807 \\
\hline 9.30922 & 16.2157 & 12.8894 \\
\hline 14.2357 & 8.72234 & 8.36498 \\
\hline 15.3128 & 8.59808 & 13.1673 \\
\hline 14.1354 & 12.5293 & 8.75003 \\
\hline 14.3615 & 12.5697 & 13.735 \\
\hline 14.6513 & 12.782 & 18.8151 \\
\hline 14.3653 & 16.3221 & 8.43517 \\
\hline 14.1246 & 15.859 & 13.7242 \\
\hline 9.25114 & 7.19249 & 10.9675 \\
\hline 8.97599 & 10.6557 & 11.3388 \\
\hline 8.71138 & 10.6648 & 16.524 \\
\hline 8.70998 & 14.3564 & 11.1693 \\
\hline 8.87832 & 14.233 & 16.4324 \\
\hline 7.92766 & 17.2414 & 10.5699 \\
\hline 12.4093 & 6.87454 & 10.9024 \\
\hline 12.4345 & 10.4604 & 6.43234 \\
\hline 12.6415 & 11.0587 & 11.7438 \\
\hline $12.6 \quad 10$ & $0.5919 \quad 16$ & 6.4905 \\
\hline 12.5319 & 14.5778 & 6.4194 \\
\hline 12.5033 & 13.9749 & 11.7197 \\
\hline 12.7354 & 14.1774 & 15.8485 \\
\hline 12.5303 & 18.0377 & 11.1018 \\
\hline 16.9261 & 7.7476 & 10.605 \\
\hline 16.3056 & 10.5346 & 11.2607 \\
\hline 15.7124 & 9.83936 & 17.3125 \\
\hline 16.1076 & 14.3915 & 11.1902 \\
\hline 16.2437 & 14.4256 & 16.2716 \\
\hline 15.7045 & 17.9107 & 10.9149 \\
\hline 18.5012 & 9.18368 & 12.6204 \\
\hline 18.0181 & 12.5793 & 11.0435 \\
\hline 17.9804 & 15.9118 & 10.7813 \\
\hline 7.03084 & 9.22934 & 10.8991 \\
\hline 6.9988 & 12.3747 & 11.189 \\
\hline 6.45935 & 15.7572 & 12.6893 \\
\hline 10.9416 & 9.13048 & 11.1249 \\
\hline 10.6447 & 8.80857 & 16.4597 \\
\hline 10.2226 & 12.4915 & 6.37658 \\
\hline 10.48 & 12.5683 & 11.129 \\
\hline 10.7912 & 12.3337 & 16.0995 \\
\hline 10.9036 & 15.9905 & 10.9827 \\
\hline 10.847 & 16.1946 & 16.4012 \\
\hline 14.0923 & 8.93787 & 11.0082 \\
\hline 14.1415 & 7.96216 & 15.9245 \\
\hline 14.7382 & 12.5392 & 6.35161 \\
\hline 14.6266 & 12.4152 & 11.1438 \\
\hline 14.5363 & 12.3282 & 16.3106 \\
\hline 14.1334 & 15.9361 & 11.0733 \\
\hline 14.4278 & 16.3092 & 16.4142 \\
\hline 9.42885 & 7.21651 & 14.0375 \\
\hline 8.81712 & 10.7773 & 8.68133 \\
\hline 9.0523 & 10.9786 & 13.851 \\
\hline 8.83933 & 14.216 & 8.56625 \\
\hline 8.92641 & 13.9459 & 13.8533 \\
\hline 9.4025 & 18.7803 & 12.5885 \\
\hline 12.5112 & 7.15558 & 13.6624 \\
\hline 12.4804 & 10.6209 & 9.04072 \\
\hline 13.4292 & 10.1579 & 14.2276 \\
\hline 12.3751 & 10.4341 & 18.9378 \\
\hline 12.5411 & 14.4143 & 9.03338 \\
\hline 11.3106 & 15.6538 & 13.8747 \\
\hline 12.5794 & 14.7164 & 18.3576 \\
\hline 12.5204 & 18.0152 & 13.9148 \\
\hline
\end{tabular}

$\begin{array}{lll}10.8814 & 9.23659 & 13.7716\end{array}$

$\begin{array}{ll}12.5212 & 8.76531\end{array}$

$\begin{array}{lll}10.3632 & 12.5494 & 18.5848\end{array}$

$\begin{array}{lll}10.7346 & 16.3028 & 8.38807\end{array}$

$\begin{array}{lll}16.2157 & 12.8894\end{array}$

$\begin{array}{lll}15.3128 & 8.59808 & 13.1673\end{array}$

$\begin{array}{lll}14.1354 & 12.5293 & 8.75003\end{array}$

$\begin{array}{lll}12.5697 & 13.735\end{array}$

$\begin{array}{lll}14.3653 & 16.3221 & 8.43517\end{array}$

$\begin{array}{lll}14.1246 & 15.859 & 13.7242\end{array}$

$\begin{array}{lll}9.25114 & 7.19249 & 10.9675\end{array}$

$\begin{array}{lll}8.70998 & 14.3564 & 11.1693\end{array}$

$\begin{array}{lll}8.87832 & 14.233 & 16.4324\end{array}$

$\begin{array}{lll}7.92766 & 17.2414 & 10.5699\end{array}$

$\begin{array}{lll}12.6415 & 11.0587 & 11.7438\end{array}$

$\begin{array}{lll}12.6 & 10.5919 & 16.4905\end{array}$

$\begin{array}{lll}12.5033 & 13.9749 & 11.7197\end{array}$

$\begin{array}{lll}12.7354 & 14.1774 & 15.8485\end{array}$

$\begin{array}{lll}12.5303 & 18.0377 & 11.1018\end{array}$

$\begin{array}{lll}6.9261 & 7.7476 & 10.605\end{array}$

$\begin{array}{lll}15.7124 & 9.83936 & 17.3125\end{array}$

$\begin{array}{lll}16.1076 & 14.3915 & 11.1902\end{array}$

$\begin{array}{lll}16.2437 & 14.4256 & 16.2716\end{array}$

$\begin{array}{lll}15.7045 & 17.9107 & 10.9149\end{array}$

$\begin{array}{lll}18.5012 & 9.18368 & 12.6204\end{array}$

$\begin{array}{lll}17.0181 & 12.5793 & 11.0435 \\ 17.9804 & 15.9118 & 10.7813\end{array}$

$\begin{array}{lll}7.03084 & 9.22934 & 10.8991\end{array}$

$\begin{array}{lll}6.9988 & 12.3747 & 11.189\end{array}$

$\begin{array}{lll}6.45935 & 15.7572 & 12.6893\end{array}$

$\begin{array}{lll}10.9416 & 9.13048 & 11.1249\end{array}$

$\begin{array}{lll}10.6447 & 8.80857 & 16.4597 \\ 10.2226 & 12.4915 & 6.37658\end{array}$

$\begin{array}{lll}10.48 & 12.5683 & 11.129\end{array}$

$\begin{array}{lll}10.7912 & 12.3337 & 16.0995\end{array}$

$\begin{array}{lll}10.9036 & 15.9905 & 10.9827\end{array}$

$\begin{array}{lll}10.847 & 16.1946 & 16.4012\end{array}$

$\begin{array}{lll}14.0923 & 8.93787 & 11.0082\end{array}$

$\begin{array}{lll}14.1415 & 7.96216 & 15.9245\end{array}$

$\begin{array}{lll}14.7382 & 12.5392 & 6.35161\end{array}$

$\begin{array}{lll}14.6266 & 12.4152 & 11.1438 \\ 14.5363 & 12.3282 & 16.3106\end{array}$

$\begin{array}{lll}14.1334 & 15.9361 & 11.0733\end{array}$

$\begin{array}{lll}14.4278 & 16.3092 & 16.4142\end{array}$

$\begin{array}{lll}9.42885 & 7.21651 & 14.0375\end{array}$

$\begin{array}{lll}8.81712 & 10.7773 & 8.68133\end{array}$

$\begin{array}{lll}9.0523 & 10.9786 & 13.851\end{array}$

$\begin{array}{lll}8.92641 & 13.9459 & 13.8533\end{array}$

$\begin{array}{lll}9.4025 & 18.7803 & 12.5885\end{array}$

$\begin{array}{lll}12.5112 & 7.15558 & 13.6624\end{array}$

$\begin{array}{lll}12.4804 & 10.6209 & 9.04072\end{array}$

$\begin{array}{lll}13.4292 & 10.1579 & 14.2276\end{array}$

$\begin{array}{lll}12.5411 & 14.4143 & 9.03338\end{array}$

$\begin{array}{lll}12.5794 & 14.7164 & 18.3576\end{array}$

$\begin{array}{lll}12.5204 & 18.0152 & 13.9148\end{array}$ 


\begin{tabular}{llc}
15.6744 & 6.0752 & 12.4818 \\
16.1502 & 10.8088 & 8.5792 \\
16.3012 & 10.7967 & 14.037 \\
16.174 & 14.2864 & 8.59476 \\
16.1273 & 14.366 & 13.647 \\
15.6255 & 17.9276 & 13.9517 \\
17.1299 & 7.57573 & 12.722 \\
17.8947 & 11.0682 & 12.544 \\
17.8881 & 14.6545 & 12.3788 \\
7.20318 & 10.4901 & 12.4753 \\
7.16514 & 13.9111 & 12.6176 \\
7.74909 & 17.4508 & 12.6829 \\
10.6407 & 7.39246 & 12.4206 \\
10.7426 & 10.8398 & 7.50203 \\
10.7306 & 10.8826 & 12.4712 \\
10.7724 & 10.7694 & 17.6426 \\
10.8377 & 14.1343 & 7.40891 \\
10.5719 & 14.2944 & 12.4295 \\
10.9171 & 14.0927 & 17.4037 \\
11.0025 & 17.6153 & 12.5233 \\
14.0037 & 7.11528 & 12.2598 \\
14.1422 & 10.9063 & 7.39658 \\
14.5132 & 10.6578 & 12.5054 \\
14.1158 & 11.0144 & 18.0647 \\
14.2371 & 14.2125 & 7.47544 \\
14.3963 & 14.2218 & 12.3755 \\
14.3297 & 14.2724 & 17.3996 \\
14.3378 & 17.7029 & 12.3915 \\
8.92196 & 8.97529 & 10.0422 \\
9.02128 & 9.03067 & 14.9441 \\
8.77665 & 12.4814 & 9.86735 \\
8.57013 & 12.368 & 15.3665 \\
9.15937 & 15.9261 & 9.75465 \\
9.26538 & 15.8185 & 15.0668 \\
12.3683 & 8.5877 & 9.54772 \\
12.3726 & 8.64615 & 15.2755 \\
12.5449 & 12.5032 & 10.1683 \\
12.7349 & 12.1682 & 15.0635 \\
12.5986 & 16.4266 & 9.56476 \\
12.6856 & 16.3732 & 15.2963 \\
15.7931 & 9.09407 & 9.72665 \\
15.7732 & 8.97608 & 15.3374 \\
16.2641 & 12.5913 & 9.81075 \\
16.3134 & 12.7726 & 15.0054 \\
16.072 & 16.1312 & 9.95878 \\
16.0951 & 16.164 & 14.8319 \\
17.5109 & 11.1134 & 17.0551 \\
\hline & &
\end{tabular}

$\mathbf{O}_{80} \mathbf{Z r}_{40} \mathbf{A u}_{1}$

(ZrO bridge, Fig. 2f)

$\begin{array}{lcc}17.2104 & 7.70671 & 14.8215 \\ 18.242 & 12.6434 & 13.8486 \\ 17.9066 & 15.8325 & 14.0234 \\ 7.14576 & 9.17334 & 13.9586 \\ 6.77622 & 12.3784 & 13.9442 \\ 7.88926 & 17.2326 & 14.7862 \\ 10.5809 & 8.73127 & 8.47044 \\ 10.782 & 9.14409 & 13.7365 \\ 10.8869 & 12.5345 & 8.7334 \\ 11.2102 & 12.5758 & 13.5681 \\ 10.2889 & 12.5088 & 18.5946\end{array}$

\begin{tabular}{|c|c|c|c|c|c|}
\hline 10.774 & 16.3373 & 8.39512 & 16.0826 & 14.1189 & 13.7887 \\
\hline 9.31771 & 16.2191 & 12.8962 & 15.6479 & 17.7764 & 14.0655 \\
\hline 14.1874 & 8.68197 & 8.40313 & 17.0868 & 7.56152 & 12.7363 \\
\hline 15.2865 & 8.63983 & 13.2797 & 17.8589 & 11.069 & 12.5194 \\
\hline 14.1104 & 12.522 & 8.72351 & 17.8637 & 14.5433 & 12.4689 \\
\hline 14.0748 & 12.51 & 13.5914 & 7.17477 & 10.5169 & 12.453 \\
\hline 14.3505 & 12.4604 & 18.7328 & 7.13829 & 13.9315 & 12.5575 \\
\hline 14.4008 & 16.3185 & 8.4873 & 7.7716 & 17.4692 & 12.6686 \\
\hline 14.1165 & 15.7694 & . 13.7744 & 10.5618 & 7.35321 & 12.4251 \\
\hline 9.17656 & 7.20095 & 10.9914 & 10.7465 & 10.8446 & 7.47465 \\
\hline 8.95538 & 10.6621 & 11.3349 & 10.7144 & 10.8536 & 12.4671 \\
\hline 8.61511 & 10.6329 & 16.5104 & 10.5745 & 10.7602 & 17.5923 \\
\hline 8.70002 & 14.3883 & 11.1385 & 10.8553 & 14.1545 & 7.3872 \\
\hline 8.77405 & 14.2383 & 16.4275 & 10.5451 & 14.2919 & 12.4094 \\
\hline 7.96213 & 17.2846 & 10.5583 & 10.8425 & 14.0687 & 17.4175 \\
\hline 12.332 & 6.86454 & 11.072 & 11.0314 & 17.6007 & 12.5623 \\
\hline 12.4339 & 10.4665 & 6.3916 & 13.9512 & 7.14952 & 12.3752 \\
\hline 12.5708 & 11.0315 & 11.6154 & 14.1357 & 10.9127 & 7.36898 \\
\hline 12.3741 & 10.6931 & 16.4455 & 14.4245 & 10.6913 & 12.4737 \\
\hline 12.5596 & 14.5959 & 6.40696 & 13.8953 & 10.6839 & 17.9535 \\
\hline 12.4745 & 13.9875 & 11.6879 & 14.2476 & 14.2258 & 7.48492 \\
\hline 12.671 & 14.1037 & 15.8902 & 14.3741 & 14.1646 & 12.4154 \\
\hline 12.5808 & 18.021 & 11.1715 & 14.2554 & 14.0532 & 17.4132 \\
\hline 16.9003 & 7.72761 & 10.6361 & 14.3783 & 17.6391 & 12.4759 \\
\hline 16.255 & 10.5259 & 11.2178 & 8.88406 & 8.99456 & 10.0329 \\
\hline 17.1076 & 10.7757 & 16.2724 & 8.95122 & 8.9618 & 14.8985 \\
\hline 16.1067 & 14.3429 & 11.2743 & 8.76913 & 12.5058 & 9.83195 \\
\hline 16.2921 & 14.2542 & 16.4673 & 8.50845 & 12.3638 & 15.3296 \\
\hline 15.7608 & 17.8642 & 11.0203 & 9.18587 & 15.9637 & 9.73469 \\
\hline 18.4339 & 9.17568 & 12.6569 & 9.22376 & 15.8006 & 15.0671 \\
\hline 18.0024 & 12.5564 & 11.0511 & 12.3283 & 8.60787 & 9.58166 \\
\hline 17.9978 & 15.8367 & 10.8953 & 12.3589 & 8.74122 & 15.3105 \\
\hline 6.99495 & 9.26812 & 10.8507 & 12.5088 & 12.5215 & 10.1403 \\
\hline 6.9918 & 12.4035 & 11.1185 & 12.6376 & 12.1907 & 15.0054 \\
\hline 6.46117 & 15.7888 & 12.6461 & 12.6323 & 16.4273 & 9.60523 \\
\hline 10.8854 & 9.10367 & 11.1202 & 12.6685 & 16.3029 & 15.3388 \\
\hline 10.5894 & 8.78025 & 16.3991 & 15.7539 & 9.0639 & 9.73537 \\
\hline 10.2425 & 12.5085 & 6.35231 & 16.0422 & 9.21203 & 15.3061 \\
\hline 10.4455 & 12.5671 & 11.1052 & 16.2349 & 12.5471 & 9.81268 \\
\hline 10.6191 & 12.3746 & 16.06 & 16.5414 & 12.5985 & 15.2631 \\
\hline 10.9065 & 16.0157 & 10.9901 & 16.1056 & 16.0877 & 10.0468 \\
\hline 10.8135 & 16.1428 & 16.4229 & 16.049 & 15.9794 & 14.9247 \\
\hline 14.0684 & 8.894 & 11.0506 & 16.6656 & 10.0228 & 18.2791 \\
\hline 14.2669 & 8.85513 & 16.336 & \multirow{3}{*}{\multicolumn{3}{|c|}{$\begin{array}{l}\mathbf{O}_{\mathbf{3 2}} \mathbf{Z r}_{\mathbf{1 6}} \mathbf{A} \mathbf{u}_{\mathbf{1}} \\
\left(2 \mathrm{O}_{2 \mathrm{c}} \text { bridge, Fig. } 2 \mathrm{~g}\right)\end{array}$}} \\
\hline 14.7554 & 12.5511 & 6.34854 & & & \\
\hline $\begin{array}{l}14.586 \\
14.5539\end{array}$ & $\begin{array}{c}12.4064 \\
12.1655\end{array}$ & $\begin{array}{c}11.0996 \\
161876\end{array}$ & & & \\
\hline 14.1555 & 15.9116 & 11.1193 & & & \\
\hline 14.4117 & 16.1232 & 16.4548 & 9.7094 & 9.42343 & 10.1158 \\
\hline 9.3409 & 7.12823 & 14.0377 & 10.4244 & 11.5317 & 11.7206 \\
\hline 8.82284 & 10.7837 & 8.66254 & 10.1335 & 12.8068 & 16.3339 \\
\hline 9.01603 & 10.947 & 13.851 & 10.6367 & 16.2534 & 10.9522 \\
\hline 8.86427 & 14.2538 & 8.53786 & 14.189 & 8.77157 & 10.9486 \\
\hline 8.86184 & 13.9542 & 13.8345 & 14.314 & 12.5703 & 11.3151 \\
\hline 9.44341 & 18.7822 & 12.6102 & 14.7418 & 12.3295 & 16.458 \\
\hline 12.5963 & 7.17104 & . 13.9069 & 14.3634 & 15.5132 & 10.9065 \\
\hline 12.4545 & 10.6116 & 8.99146 & 9.45174 & 10.6599 & 14.1068 \\
\hline 13.1215 & 10.2024 & 14.0714 & 8.73193 & 14.3787 & 13.9771 \\
\hline 12.1841 & 10.1625 & 18.8408 & 12.7513 & 10.2252 & 8.64696 \\
\hline 12.5452 & 14.4308 & 9.01155 & 12.4325 & 10.6859 & 13.7794 \\
\hline 11.3023 & 15.6116 & 13.8978 & 12.2493 & 14.8302 & 8.65127 \\
\hline 12.5339 & 14.6203 & 18.3727 & 13.4559 & $14.59 \quad 1$ & 13.2935 \\
\hline 12.5467 & 17.9578 & 13.9837 & 16.179 & 10.8229 & 14.1161 \\
\hline 15.6126 & 6.08953 & 12.5417 & 15.5209 & 15.3452 & 14.8864 \\
\hline 16.1136 & 10.7694 & 8.55949 & 10.9636 & 8.42031 & 12.5772 \\
\hline 16.1846 & 10.9212 & 13.8767 & 10.3788 & 12.5599 & 8.94054 \\
\hline 16.1754 & 14.2662 & 8.64994 & 11.0724 & 12.976 & 13.919 \\
\hline
\end{tabular}




$\begin{array}{lll}10.9032 & 16.0229 & 13.679 \\ 14.0943 & 8.74 & 14.159 \\ 14.5809 & 12.4165 & 8.73823 \\ 14.224 & 12.3898 & 13.9176 \\ 13.9217 & 17.2008 & 12.8166 \\ 7.80282 & 11.0581 & 12.1347 \\ 8.96717 & 14.0827 & 11.2482 \\ 12.6358 & 10.7456 & 11.1697 \\ 12.5122 & 10.4365 & 16.3756 \\ 12.0332 & 13.9102 & 11.0854 \\ 12.3803 & 14.714 & 16.0324 \\ 16.2622 & 10.8937 & 10.8998 \\ 16.5805 & 14.0415 & 12.4523 \\ 11.1144 & 10.7741 & 9.59478 \\ 10.9602 & 11.238 & 15.3104 \\ 10.5971 & 14.3875 & 9.6843 \\ 10.5552 & 14.4545 & 15.2466 \\ 14.4161 & 10.618 & 9.9281 \\ 14.367 & 10.5547 & 15.0339 \\ 13.8143 & 13.9626 & 9.6692 \\ 14.1514 & 13.9886 & 15.368 \\ 9.42308 & 9.65295 & 12.0908 \\ 8.9243 & 12.5266 & 12.7682 \\ 9.24557 & 15.7546 & 12.4492 \\ 12.9503 & 8.9193 & 12.5029 \\ 12.5887 & 12.4044 & 12.5076 \\ 12.4792 & 16.0557 & 12.1486 \\ 16.0696 & 12.0536 & 12.492 \\ 15.3371 & 15.5929 & 12.8685\end{array}$

\begin{tabular}{lcc}
13.2723 & 11.8518 & 17.9832 \\
\hline \multicolumn{3}{c}{$\mathbf{O}_{32} \mathbf{Z r}_{\mathbf{1 6}} \mathbf{A u}_{\mathbf{1}}$} \\
$\mathbf{O}_{2 \mathrm{c}}$ top, Fig. $\left.2 \mathrm{~h}\right)$ \\
\multicolumn{3}{c}{} \\
9.78467 & 9.38896 & 10.1556 \\
10.4809 & 11.5086 & 11.7485 \\
10.24 & 12.7481 & 16.382 \\
10.5126 & 16.2388 & 11.0211 \\
14.3296 & 8.91232 & 10.8383 \\
14.3143 & 12.6994 & 11.1935 \\
14.7842 & 12.3484 & 16.3047 \\
14.2323 & 15.6322 & 10.8407 \\
9.5727 & 10.5802 & 14.1485 \\
8.75372 & 14.2911 & 14.0721 \\
12.7366 & 10.3201 & 8.60031 \\
12.5372 & 10.7497 & 13.7507 \\
12.077 & 14.8943 & 8.6366 \\
13.4614 & 14.5988 & 13.238 \\
16.4461 & 11.0499 & 14.0342 \\
15.598 & 15.3843 & 14.7579 \\
11.1816 & 8.43807 & 12.5649 \\
10.2911 & 12.5582 & 8.98337 \\
11.1251 & 12.9541 & 13.9352 \\
10.8709 & 15.9867 & 13.721 \\
14.3879 & 8.90524 & 14.0329 \\
14.506 & 12.5695 & 8.62186 \\
14.4004 & 12.3933 & 13.7634 \\
13.8232 & 17.2439 & 12.8221
\end{tabular}

\begin{tabular}{lll}
7.89116 & 10.9514 & 12.2171 \\
8.93331 & 14.0221 & 11.3503 \\
12.7074 & 10.8197 & 11.1304 \\
12.6537 & 10.3882 & 16.2924 \\
11.9835 & 13.9425 & 11.0712 \\
12.4773 & 14.6747 & 16.0021 \\
16.3135 & 11.0849 & 10.7273 \\
16.5779 & 14.2249 & 12.2124 \\
11.1108 & 10.8025 & 9.61582 \\
11.0835 & 11.1925 & 15.3522 \\
10.4816 & 14.3872 & 9.73095 \\
10.6313 & 14.399 & 15.3091 \\
14.4227 & 10.762 & 9.80472 \\
14.3363 & 10.6654 & 15.1046 \\
13.7096 & 14.0839 & 9.58826 \\
14.2636 & 13.9847 & 15.3102 \\
9.55806 & 9.59458 & 12.1463 \\
8.97928 & 12.4412 & 12.8603 \\
9.18144 & 15.686 & 12.5579 \\
13.1445 & 9.02152 & 12.4436 \\
12.6433 & 12.4632 & 12.4859 \\
12.3971 & 16.0687 & 12.1532 \\
16.1091 & 12.2569 & 12.2935 \\
15.2862 & 15.7047 & 12.7871 \\
17.7904 & 9.6194 & 13.7851 \\
\hline
\end{tabular}

$\begin{array}{lll}14.4004 & 12.3933 & 13.7634\end{array}$ 